\title{
Phase noise in a Colpitts oscillator
}

\author{
Andrew Allison ${ }^{a}$ and Derek Abbott ${ }^{a}$ \\ ${ }^{a}$ Centre for Biomedical Engineering (CBME) and School of Electrical \& Electronic \\ Engineering, The University of Adelaide, SA 5005, Australia.
}

\begin{abstract}
We apply the technique of the calculus of stochastic differential equations to the problem of noise in an electronic circuit with positive feedback. We argue that this is a very natural approach to the more general problem of noise in electronic circuits of all types. We apply the standard small-signal analysis to the circuit, incorporating the standard high-frequency small-signal model for the field effect transistor. This allows us to derive a statevariable model for the system, which is essentially a coupled system of Ordinary Differential Equations. If we then incorporate a standard noise model for the field effect transistor, we obtain a coupled system of Stochastic Differential Equations, or SDEs. We apply the stochastic differential calculus of Itõ to this problem and compare the results with simulations. We examine the dependence of phase-noise on the system parameters. We also simulate the case where the oscillations become large and use this to investigate the limits of the small-signal approximation.
\end{abstract}

Keywords: state variable, stochastic differential equations, Colpitts oscillator, phase noise

\section{INTRODUCTION}

The traditional approach to thermal noise in circuits can be traced back to Johnson. ${ }^{1}$ Over the years, a number of empirical techniques have been developed to estimate noise in active circuits that filter the Johnson noise. We argue that an important issue in this analysis is the way in which one represents white noise. One surprising aspect of white noise is that it cannot be constructed as an ordinary random process. True Brownian motion, if it could be constructed, would contain gaps or jumps. Also, true Brownian motion would have infinite bandwidth and would require infinite power to construct. Fortunately there is a well defined random process that can be used to model white noise. This is known as the Wiener process or Brownian motion. We can say that an infinitesimal increment of Brownian motion, $d B$, has the same measure as white noise, $Z$, over an infinitesimal increment of time, $d t$. We can represent this symbolically as $d B=Z d t$. The theory of stochastic differential equations $^{2}$ establishes the existence of integration with respect to white noise, over a finite time interval $\left[T_{1}, T_{2}\right]$, $I=\int_{T_{1}}^{T_{2}} f(t) d B$. We argue that the calculus of stochastic differential equations is the natural tool to use to analyse noise in electronic circuits. Further, we show that in many cases we do not actually have to solve the SDEs directly in order to evaluate parameters of interest, such as noise power. It is possible to derive an ODE from the SDE to describe the evolution of the parameters in time.

\section{A ONE-DIMENSIONAL EXAMPLE}

We consider the well known case of a resistor in parallel with a capacitor. ${ }^{3}$ This is shown in Figure 1 . We expect to find a mean-square voltage across the capacitor of, $\left\langle V^{2}\right\rangle=\frac{k T}{C}$. This is clear if we regard the voltage across the capacitor as a single degree of freedom and apply the principle of equipartition of energy, $\left\langle\frac{1}{2} C V^{2}\right\rangle=\frac{1}{2} k T$.

We represent the resistor using a Norton model of an ideal resistor, $R$, in parallel with a noise source, denoted by $i_{0} d B$ where $i_{0}=\sqrt{2 k T / R}$ and $d B$ is an infinitesimal increment of Brownian motion. In some of the literature this is represented by $d W$ which is an infinitesimal increment of the Wiener process. These two notations are mathematically equivalent.

Further author information: (Send correspondence to A. Allison, School of Electrical and Electronic Engineering, The University of Adelaide, SA 5005, Australia.) E-mail: aallison@eleceng.adelaide.edu.au, Telephone:+61 883035283 


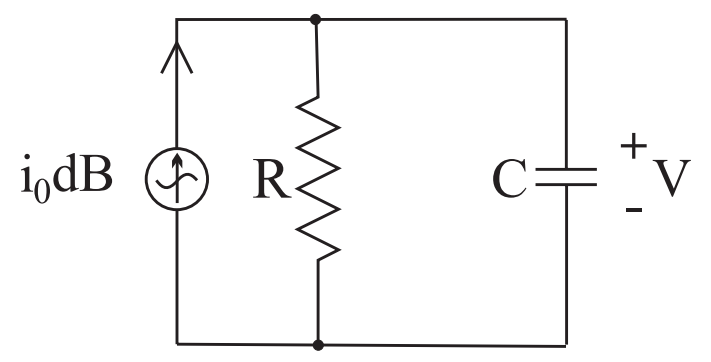

Figure 1. $R C$ parallel circuit, with resistor, $R$, noise current source, $i_{o} d B$, and capacitor, $C$.

The mean square current varies linearly with $k T$ and the coefficient of $k T$ is 2 , not 4 as it is in some references. The choice of $2 k T$ is required to make the results consistent with a definition of frequency used for complex exponentials, rather than sinusoids. It is consistent with widely used definitions of power spectral density and autocorrelation functions. ${ }^{4-7}$ It includes positive and negative frequencies and is called the two-sided power spectrum. ${ }^{7}$

We consider the nodal equation for the circuit. We require $\sum d Q=0$. For the capacitor, we have $d Q=C d V$, where $C$ is the capacitance and $d V$ is an infinitesimal increment of voltage. For the resistor, we have $d Q=\frac{V}{R} d t$ and for the equivalent noise source in the resistor, we have $d Q=i_{0} d B$, as discussed above. The nodal equation then becomes

$$
C d V+\frac{1}{R} V d t=i_{0} d B
$$

which is the SDE for this system. This can be re-written as an SDE in the narrow sense:

$$
d V=\frac{-1}{R C} V d t+\frac{i_{0}}{C} d B
$$

This is of the form

$$
d V=\alpha(t) V d t+\beta(t) d B
$$

where $\alpha(t)=\alpha=-1 /(R C)$ and $\beta(t)=\beta=+i_{0} / C=\sqrt{\left(2 k t /\left(R C^{2}\right)\right.}$. If we define the expected value of $V$ as $\mu=E[V]$ and the variance as $\nu=E\left[(V-\mu)^{2}\right]$ then it can be shown ${ }^{8}$ that

$$
\frac{d \nu(t)}{d t}=2 \alpha(t) \nu(t)+\beta(t)^{2}
$$

If we consider the steady-state situation after all transients have decayed then we have $\frac{d \nu(t)}{d t}=0$, which implies that

$$
\nu=E\left[(V-\mu)^{2}\right]=-\frac{\beta(t)^{2}}{2 \alpha(t)}=\frac{k t}{C},
$$

which is the classical result. Note that we did not solve the SDE but only used it to derive an ODE. We did not solve the ODE but only used it to derive an algebraic equation, which we then used to solve for the steady state value of the noise power. We believe that this simple and systematic method is general and should find wide application in the analysis of noise in circuits. In summary, we proceed from the circuit to a nodal, or mesh, equations to an SDE to an ODE to an algebraic equation and then obtain estimates of noise power.

\section{NOISE MODELS FOR THE JFET}

The noise model that we use here, shown in Figure 2 is the one used by Abbott et al. and is essentially a van der Ziel model with all noise sources referred to the input. For a JFET, the gate currents are limited by reverse biased $\mathrm{PN}$ junctions. We regard the gate leakage current as negligible and have not included it in the model. 


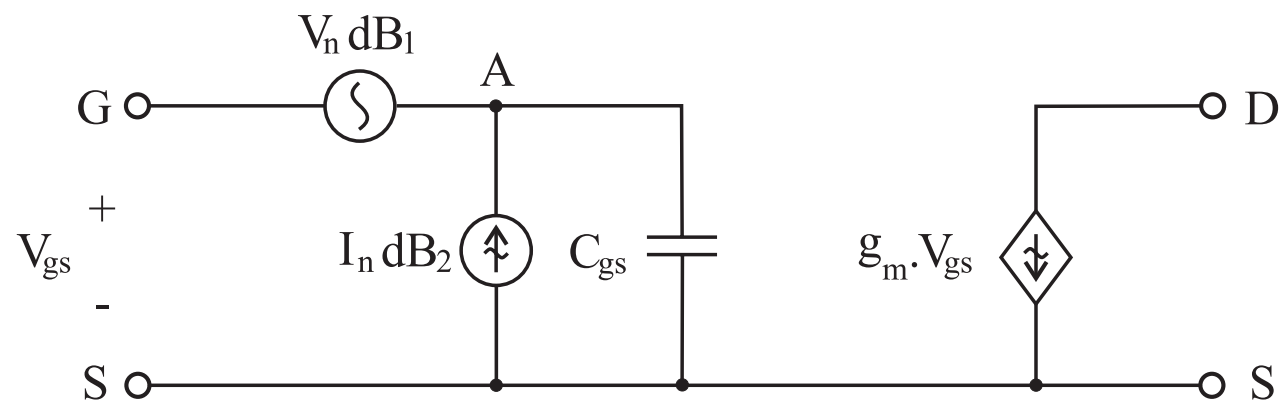

Figure 2. Noise model for a JFET. This is basically a van der Ziel model with all noise sources referred to the input. The gate to source capacitance is represented by $C_{g s}$. This model includes the standard noise-free small signal model for a JFET. The amplifying effect of the JFET is represented by the dependent current source, $g_{m} \cdot V_{g s}$. The noise is represented by two sources at the input, $V_{n} \cdot d B_{1}$ and $I_{n} \cdot d B_{2}$ where $d B_{1}$ and $d B_{2}$ are infinitesimal increments of Brownian motion.

\section{A SIMPLE JFET CIRCUIT}

We use a very simple version of the Colpitts oscillator with a FET as the amplifying element. This is shown in Figure 3. In the Colpitts topology, the split capacitor, $C_{1}$ and $C_{2}$, allows for a feedback path with high impedance and voltage amplification.

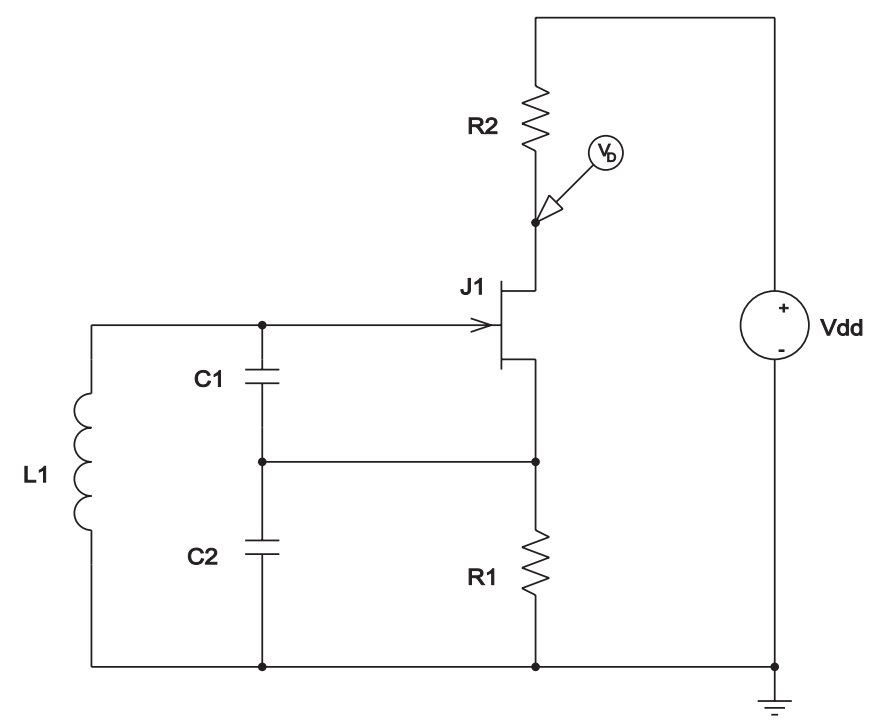

Figure 3. Standard, large-signal, schematic circuit diagram for a Colpitts oscillator using a JFET as the amplifying element. There are noise sources in $R_{1}, R_{2}$ and $J_{1}$.

\section{ANALYSIS OF THE JFET CIRCUIT}

If we analyse the circuit in Figure 3, using small-signal technique, and insert the noise model from Figure 2 then we obtain the complete small signal noise model for the Colpitts oscillator, shown in Figure 4. If the circuit did not have noise sources then ordinary mesh and nodal analysis could be performed and we obtain a standard state-variable model. ${ }^{9}$ With the presence of noise sources, Kirchhoff's current law takes the form: $\sum d Q=\sum I d t=0$ where the contribution from a noise current source would be $d Q=I_{n} d B$. The increment of accumulated charge (electric flux) at a node is always zero. With the presence of noise sources, Kirchhoff's 


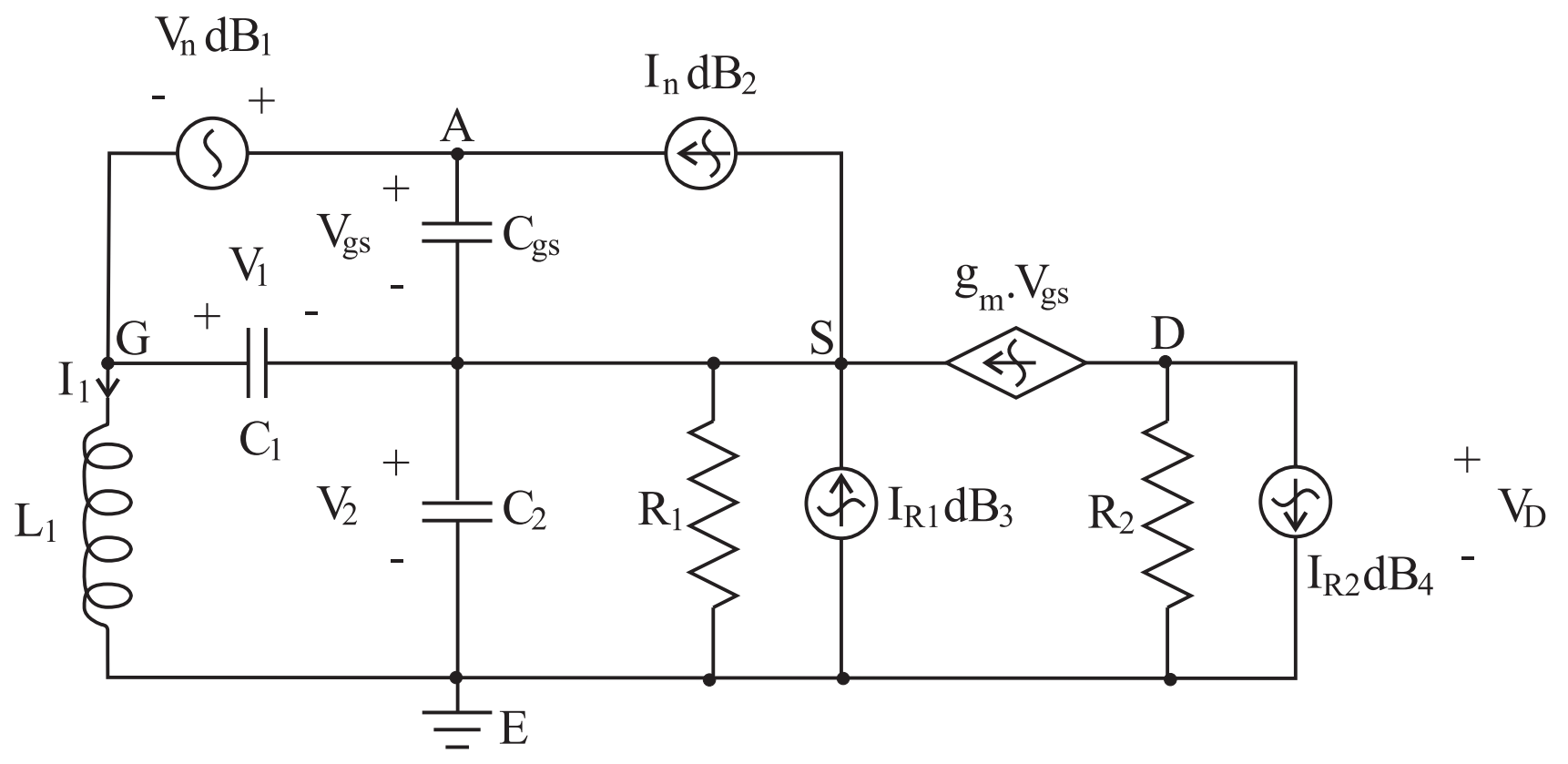

Figure 4. Small signal equivalent circuit, including the van der Ziel noise model. The FET is mapped into the equivalent circuit with Source, $S$, Gate, $G$, Drain $D$. The earth is represented by $E$. The circuit has four energy storage elements, $C_{g s}, C_{1}, C_{2}$ and $L_{1}$. There are four corresponding state variables, $V_{g s}, V_{1}, V_{2}$ and $I_{1}$. Further analysis shows that only three of these are independent. There are four noise sources, $V_{n} \cdot d B_{1}, I_{n} \cdot d B_{2}, I_{R 1} \cdot d B_{3}$ and $I_{R 2} \cdot d B_{4}$. The nominal output is the drain voltage, $V_{D}$.

voltage law takes the form: $\sum d \Phi=\sum V d t=0$ where the contribution from a noise voltage source would be $d \Phi=V_{n} d B$. The increment of accumulated magnetic flux around a mesh is always zero.

We obtain a mesh equation for the mesh including the Gate $G$ and the node $A$,

$$
V_{1} d t-V_{g s} d t+V_{n} d B_{1}=0
$$

which indicates that the state-variables, $V_{1}$ and $V_{g s}$ are not independent. We can obtain a mesh equation including $L_{1}, C_{1}$ and $C_{2}$,

$$
d I_{1}=\frac{1}{L_{1}} \cdot V_{1} d t+\frac{1}{L_{1}} \cdot V_{2} d t
$$

The current through the noise source, $V_{n} d B_{1}$ is undetermined but we can regard $G$ and $A$ as an enlarged node and write

$$
I_{1} d t+C_{1} d V_{1}+C_{g s} d V_{g s}-I_{n} d B_{2}=0 .
$$

Finally, the source is simply a very large node

$$
C_{1} d V_{1}-C_{2} d V_{2}+C_{g s} d V_{g s}=\frac{V_{2}}{R_{1}} d t-g_{m} V_{g s} d t+I_{n} d B_{2}-I_{R 1} d B_{3}
$$

Equations 6, 7, 8 and 9 are the equations of state for this system.

The nominal output of the circuit is the drain voltage, $V_{D}$. This can be expressed with an output equation,

$$
V_{D} d t=-R_{2} g_{m} V_{g s} d t-I_{R 2} R_{2} d B_{4}
$$

This completes the formulation of the circuit. 


\section{OPEN QUESTIONS}

The problem has been formulated but not yet completely solved. The plan is to analyse the noise power implied by Equations 6, 7, 8, 9 and 10 using the technique impled by Equation 5 and to check the result using standard numerical simulation techniques. ${ }^{10,11}$

\section{REFERENCES}

1. J. B. Johnson Phys. Rev. 32(97), 1928.

2. B. Øksendal, Stochastic Differential Equations, Springer, Berlin, 1998.

3. R. P. Feynman, R. B. Leighton, and M. Sands, The Feynman Lectures on Physics, vol. 1, Addison-Wesley, Reading, Massachusetts, 1963.

4. R. N. Bracewell, The Fourier Transform and its Applications, McGraw-Hill, third ed., 1965, 3rd ed. 2000.

5. R. P. Yates and D. J. Goodman, Probability and Stochastic Procresses, John Wiley \&Sons inc., New York, 1999.

6. P. Z. Peebles, Probability, Random Variables, and Random Signal Principles, Mc Graw Hill, Singapore, 2001.

7. Design of Analog CMOS Integrated Circuits, McGraw Hill, Boston, 2001.

8. J. A. Gubner, The Control Handbook, ch. 60, Stochastic Differential Equations, pp. 1067-1078. CRC Press and IEEE Press, 1996.

9. P. M. DeRusso, R. J. Roy, and C. M. Close, State Variables for Engineers, John Wiley \& Sons inc., New York, 1965.

10. P. E. Kloeden and E. Platen, Numerical Solution of Stochastic Differential Equations, Springer-Verlag, Berlin, 1992.

11. C. Penski, "A new numerical method for sdes and its application in circuit simulation," Journal of Computational and Applied Mathematics 115(1-2), pp. 461-70, 2000. 